\title{
Positron lifetime measurements on neutron-irradiated InP crystals
}

\author{
F. J. Navarro a) and L. C. Damonte ${ }^{\text {b) }}$ \\ Instituto de Física Corpuscular (CSIC-Universitat de València), 46100 Burjassot, Spain \\ B. Marí \\ Departament de Física Aplicada, Universitat Politècnica de València, E-46071 València, Spain \\ J. L. Ferrero \\ Departament de Física Atómica, Molecular y Nuclear, Facultad de Física, Universitat de València, \\ 46100 Burjassot, Spain
}

(Received 19 December 1995; accepted for publication 22 February 1996)

\begin{abstract}
Neutron-irradiated InP single crystals have been investigated by positron-lifetime measurements. The samples were irradiated with thermal neutrons at different fluences yielding concentrations for Sn-transmuted atoms between $2 \times 10^{15}$ and $2 \times 10^{18} \mathrm{~cm}^{-3}$. The lifetime spectra have been analyzed into one exponential decay component. The mean lifetimes show a monotonous increase with the irradiation dose from 246 to 282 ps. The increase in the lifetime has been associated to a defect containing an Indium vacancy. Thermal annealing at $550{ }^{\circ} \mathrm{C}$ reduces the lifetime until values closed to those obtained for the as-grown and conventionally doped InP crystals. (C) 1996 American Institute of Physics. [S0021-8979(96)03711-5]
\end{abstract}

\section{INTRODUCTION}

Positron annihilation spectroscopy (PAS) has become a valuable tool to study the properties in solids. ${ }^{1}$ In particular, positron techniques have demonstrated its capability on analyze the defect structure on semiconductor materials. ${ }^{2,3}$ At present, the positron annihilation is successfully applied to investigate the structure of the native defects in compound III-V semiconductors. ${ }^{4-6}$ However, few works have been reported in InP, which have attracted much attention because it is one of the most promising materials for the development of optoelectronics.

In this material, the positron lifetime technique has been applied to investigate the nature of the native defects in asgrown InP. ${ }^{7,8}$ It is found that the experimental bulk positron lifetime values published vary from 235 to 247 ps. Recently, Bretagnon et al. ${ }^{8}$ have reported a systematic study of grown-in defects in crystals doped with different dopants and carrier concentrations. These authors have resolved a second lifetime, between 263 and 272 ps which has been attributed to positrons annihilating at indium vacancy-impurity related defects.

Neutron-transmutation doping (NTD) is a convenient method for semiconductor doping purposes when a precise amount and an homogeneous distribution of the impurities are required. NTD relies on the capture of thermal neutrons by the host lattice nuclei. The process depends on the capture cross-sections and the natural abundance of each isotope. It has been shown that InP can be effectively doped by neutron transmutation, yielding a precise concentration of doping atoms which act as donors and control the electron concentration. ${ }^{9,10}$

The main impurity introduced in InP by NTD is Sn, which comes from the transmutation of the most naturally

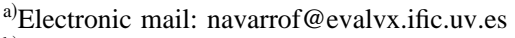

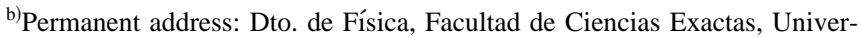
sidad Nacional de La Plata, 1900 La Plata, Argentina.
}

abundant ${ }^{115}$ In isotope $(95.7 \%)$ according the following reaction:

$$
{ }^{115} \operatorname{In}(n, \gamma){ }^{116} \mathrm{In} \rightarrow{ }^{116} \mathrm{Sn}+\beta^{-} .
$$

${ }^{115}$ In has a high neutron capture cross section $\left(\sigma_{c}=199 \mathrm{~b}\right)$. The rest of the isotopes of InP have the following neutron capture cross-sections and natural abundance: $10.7 \mathrm{~b}$ and $4.3 \%$ for ${ }^{113}$ In and $0.19 \mathrm{~b}$ and $100 \%$ of abundance for ${ }^{31} \mathrm{P}$.

${ }^{113}$ In transmute mainly in $\mathrm{Sn}$ and ${ }^{31} \mathrm{P}$ into $S$. Among all the nuclear reactions, $99.9 \%$ transmute $\mathrm{In}$ to $\mathrm{Sn}$ and about $0.1 \%$ transmute $\mathrm{P}$ into $S$ atoms. The values of neutron capture cross-sections and natural abundances were extracted from. ${ }^{11}$

During the NTD process some lattice defects are created due to the $\gamma$ and $\beta$ rays produced in the nuclear reaccions and the fast neutrons which unavoidable accompany the thermal neutron flux and cannot be completely eliminated. Therefore, a suitable annealing step has to be performed in order to recover the crystal lattice. In the case of InP, annealing at $550{ }^{\circ} \mathrm{C}$ is enough to electrically activate the doping impurities. This has been achieved using both classical (10 min) and rapid (10 s) thermal annealing methods. ${ }^{9}$

We present here lifetime positron measurements in unirradiated and neutron-irradiated $\mathrm{InP}$ single crystals and the evolution of their lifetime parameters as a function of the calculated Sn concentration. The main purpose of this work is to study the nature of radiation induced defects affecting the positron annihilation lifetime and their recover after thermal annealing.

\section{EXPERIMENT}

The starting material used were undoped $n$-type LECgrown InP, with a free carrier density of about $(2-4) \times 10^{15}$ $\mathrm{cm}^{-3}$ at room temperature. Samples of approximated dimensions $7 \times 7 \mathrm{~mm}^{2}$ and $350 \mu \mathrm{m}$ thick were extracted from the same wafer and subsequently irradiated within a low flux nuclear reactor with several doses of thermal neutrons in order to obtain additional doping concentrations ranging 
TABLE I. Irradiation conditions and expected $\mathrm{Sn}$ concentrations in the samples used in this work. Samples 10 and 11 were annealed at $550{ }^{\circ} \mathrm{C}$ with a residence time of $10 \mathrm{~min}$ and then measured at room temperature.

\begin{tabular}{|c|c|c|c|c|}
\hline \multirow[b]{2}{*}{ Ref. } & \multirow[b]{2}{*}{ Sample } & \multicolumn{2}{|c|}{ Irradiation parameters } & \multirow[b]{2}{*}{ Observations } \\
\hline & & $\begin{array}{c}\text { Flux } \\
{\left[\mathrm{cm}^{-1} \mathrm{~s}^{-1}\right]}\end{array}$ & $\begin{array}{l}\text { Fluence } \\
{\left[\mathrm{cm}^{-2}\right]}\end{array}$ & \\
\hline (1) & as-grown & & & \\
\hline (2) & $\mathrm{NTD}:[\mathrm{Sn}]=2 \times 10^{15}$ & $10^{12}$ & $5.30 \times 10^{14}$ & \\
\hline (3) & $\mathrm{NTD}:[\mathrm{Sn}]=4 \times 10^{15}$ & $10^{12}$ & $1.06 \times 10^{15}$ & \\
\hline (4) & $\mathrm{NTD}:[\mathrm{Sn}]=1 \times 10^{16}$ & $10^{12}$ & $2.65 \times 10^{15}$ & \\
\hline (5) & $\mathrm{NTD}:[\mathrm{Sn}]=3 \times 10^{16}$ & $10^{12}$ & $7.95 \times 10^{15}$ & \\
\hline (6) & $\mathrm{NTD}:[\mathrm{Sn}]=1 \times 10^{17}$ & $10^{12}$ & $2.65 \times 10^{16}$ & \\
\hline (7) & $\mathrm{NTD}:[\mathrm{Sn}]=3 \times 10^{17}$ & $4.4 \times 10^{11}$ & $7.95 \times 10^{16}$ & \\
\hline (8) & $\mathrm{NTD}:[\mathrm{Sn}]=1 \times 10^{18}$ & $4.7 \times 10^{11}$ & $2.65 \times 10^{17}$ & \\
\hline (9) & $\mathrm{NTD}:[\mathrm{Sn}]=2 \times 10^{18}$ & $4.4 \times 10^{11}$ & $5.30 \times 10^{17}$ & \\
\hline (10) & $\mathrm{NTD}:[\mathrm{Sn}]=10^{17}$ & $10^{12}$ & $2.65 \times 10^{16}$ & $550^{\circ} \mathrm{C}, 10 \mathrm{~min}$ \\
\hline (11) & $\mathrm{NTD}:[\mathrm{Sn}]=3 \times 10^{17}$ & $4.4 \times 10^{11}$ & $7.95 \times 10^{16}$ & $550^{\circ} \mathrm{C}, 10 \mathrm{~min}$ \\
\hline (12) & $\mathrm{NTD}:[\mathrm{Sn}]=1 \times 10^{18}$ & $4.7 \times 10^{11}$ & $2.65 \times 10^{17}$ & $550{ }^{\circ} \mathrm{C}, 10 \mathrm{~min}$ \\
\hline (13) & $\begin{array}{l}\text { Conven. Fe-Doped } \\
{[\mathrm{Fe}]=(3-4) \times 10^{16}}\end{array}$ & & & \\
\hline (14) & $\begin{array}{l}\text { Conven. Sn-Doped } \\
{[\mathrm{Sn}]=4.8 \times 10^{17}}\end{array}$ & & & \\
\hline
\end{tabular}

from $2 \times 10^{15}$ to $2 \times 10^{18} \mathrm{~cm}^{-3}$. Due to the low neutron flux used $\left(\phi \leqslant 10^{12} \mathrm{n} / \mathrm{cm} \mathrm{s}\right)$, the samples remained at room temperature during the irradiation. The thermal neutron flux and fluences as well as the expected Sn concentrations are listed in Table I.

Apart from the irradiated and the as-grown samples, two others InP samples were measured: Conventionally Fe and Sn doped with a free carrier concentration at room temperature of about $10^{8} \mathrm{~cm}^{-3}$ and $4.8 \times 10^{17} \mathrm{~cm}^{-3}$, respectively.

The thermal annealing were performed at $550{ }^{\circ} \mathrm{C}$ during 10 min under an argon gas flow at ambient pressure.

A conventional fast-fast lifetime equipment with two $\mathrm{BaF}_{2}$ detectors has been used. The time resolution [full width at half-maximum (FWHM)] was 250 ps for ${ }^{60} \mathrm{Co}$ and the time calibration was $18.7 \mathrm{ps} /$ channel. The source, ${ }^{22} \mathrm{NaCl}$ (about $10 \mu \mathrm{Ci}$ ) sealed in a kapton foil $\left(1.42 \mathrm{~g} / \mathrm{cm}^{3}\right)$, is sandwiched between two samples. Positron lifetime spectra were recorded at room temperature and the data were analyzed with POSITRONFIT. ${ }^{13,14}$

The lifetime spectra contained no less than $2 \times 10^{6}$ counts and a source correction with two components was used in the analysis: The first one of 382 ps with $12 \%$ intensity (kapton foil contribution), and the second one of 800 ps with $1 \%$ intensity. Each measurement was repeated at least three times.

\section{RESULTS AND DISCUSSION}

The time spectrum of the undoped sample (sample 1) has been fitted by one exponential decay component. The obtained value $(246 \pm 2 \mathrm{ps})$ agrees with those previously reported by Dlubek et al. ${ }^{7}$ and Puska et al. ${ }^{15}$ for this material. A two terms fit, as suggested by other authors (Bretagnon et $\left.a l .{ }^{8}\right)$, yields a very low value for $\mathrm{I}_{2}(<1 \%)$ and does not substantially improve the variance of the fit. The lifetime values found for $n$-type ( $\mathrm{Sn}$-doped) and semi-insulating (Fe-

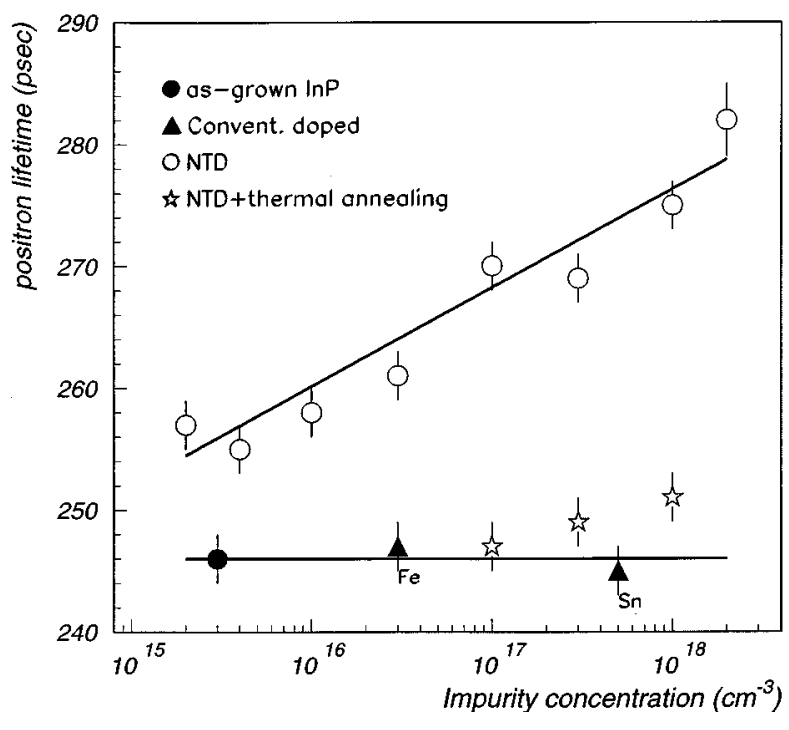

FIG. 1. Lifetime parameters versus the Impurity concentration. Empty symbols - correspond to samples in the as-irradiated condition. TrianglesCrystals conventionally doped with Fe $\left(3 \times 10^{16} \mathrm{~cm}^{-3}\right)$ and $\mathrm{Sn}\left(4.8 \times 10^{17}\right.$ $\left.\mathrm{cm}^{-3}\right)$. Full circle-As-grown material. Stars-Samples annealed at $550^{\circ} \mathrm{C}$ during $10 \mathrm{~min}$ and then measured at room temperature. The horizontal dashed line represents the mean value obtained in undoped and conventionally doped samples.

doped) samples were $245 \pm 2$ and $247 \pm 2$ ps, respectively. These results are closed to those obtained in the undoped samples.

The results of the experiments have been represented in Fig. 1. Empty symbols correspond to samples in the asirradiated condition and the measures are plotted at their Snexpected concentrations. Full symbols correspond to the asgrown and conventionally doped samples. In the $x$ axis the impurity concentration has been represented. Error bars are include when they are larger than the symbol size.

The full circle corresponds to the as-grown material used as starting material for the neutron transmutation doping experiments. This material was $n$ type with $n(300$ $\mathrm{K}) \sim(2-4) \times 10^{15} \mathrm{~cm}^{-3}$. The room temperature electron concentration comes from the existence of residual native donors in the as-grown material. The measured lifetime value is 246 ps. The semi-insulating sample (Fe-doped) had a resistivity value in the range of $\sim 10^{7} \Omega \mathrm{cm}$. The semi-insulating behavior is due to the presence of $\mathrm{Fe}$ atoms which act as acceptors and compensate the residual donors. The [Fe] value is about $3 \times 10^{16} \mathrm{~cm}^{-3}$ and the lifetime value is $247 \pm 2$ ps. The Sn-doped sample is $n$ type with an electron concentration measured by Hall effect of $n(300 \mathrm{~K}) \sim 4.8 \times 10^{17}$ $\mathrm{cm}^{-3}$. The lifetime value found is $245 \pm 2 \mathrm{ps}$.

The horizontal solid line represents the average value of lifetime obtained in undoped and conventionally doped InP samples. It can be conclude that the nature and concentration of dopants does not affect the positron annihilation in doped samples, in agreement with the results reported by Bretagnon et $a .^{8}$ for InP with different types of dopants (Fe, $\mathrm{Zn}, \mathrm{S}, \mathrm{Si}$ ) and carrier concentrations. This result means that the positron lifetime in InP does not depend of the Fermi level position. Unlike, in other III-V materials, as for example GaAs, the lifetime seems to be a function of the Fermi 
level. ${ }^{16}$ This difference might be due to the fact that in GaAs the Fermi level is controlled by the midgap level EL2. This level is an intrinsic defect which can trap positrons depending on it state of charge.

The open circles in Fig. 1 correspond to the irradiated samples. In this case, the $x$ axis represents the expected Sn concentration calculated from the irradiation dose. The tin concentration can be written as ${ }^{9}$

$$
[\mathrm{Sn}]=(\phi t) \sum_{i} n_{i} \sigma_{c}^{i}
$$

where $(\phi t)$ is the thermal neutron fluence, and $n_{i}$ and $\sigma_{c}^{i}$ are the concentration and capture cross-section of the In $i$ th isotope, respectively. For InP, the neutron absorption coefficient, $\Sigma_{i} n_{i} \sigma_{c}^{i}$, reaches a value of $3.77 \mathrm{~cm}^{-1}$.

The lifetime spectra were analyzed with one component. It is found that the neutron irradiated samples yielded a lifetime longer than the measured one on undoped InP. The lifetime values show a monotonous increase with the thermal neutron dose and vary from $257 \pm 2 \mathrm{ps}\left(2 \times 10^{15} \mathrm{~cm}^{-3}\right)$ to $282 \pm 3 \mathrm{ps}\left(2 \times 10^{18} \mathrm{~cm}^{-3}\right)$. This effect shows that certain lattice defects induced by thermal neutrons act as effective positron traps.

In our case, the lifetime obtained from the singlecomponent analyses should be interpreted as a weighted mean lifetime of positrons annihilating in the bulk and at the induced defects. According to the positron trapping model this mean lifetime parameter, $\bar{\tau}$, is a linear superposition of their values in the bulk, $\tau_{b}$, and at the positron traps, $\tau_{d}$ :

$$
\bar{\tau}=\left(1-\alpha_{d}\right) \tau_{b}+\alpha_{d} \tau_{d},
$$

where $\alpha_{d}$ is the fraction of positrons annihilating at vacancy defects. These defects should be produced by the $\gamma$ and $\beta^{-}$ particles involved in the doping process. In this process, vacancies can be formed in both sublattices. The positrons can be trapped by one or both of them and for that, $\tau_{d}$ would be a mixture of two contributions: annihilations in $V_{\text {In }}-\mathrm{Sn}_{\text {In }}$ (In vacancy-Sn interstitial) and in $V_{P}-S_{\text {In }}(P$ vacancy- $S$ interstitial).

Theoretical calculations ${ }^{15}$ suggest that the increase in lifetime from the bulk lifetime is $50 \mathrm{ps}$ for $V_{\text {In }}$ and 28 ps for $V_{P}$. Experimentally, we obtain an increase in $\bar{\tau}$ relative to the bulk lifetime close to $40 \mathrm{ps}\left([\mathrm{Sn}]=2 \times 10^{18} \mathrm{~cm}^{-3}\right)$. By comparing both results, we can conclude that the increase observed in $\bar{\tau}$ is more probably due to positrons trapped at Indium than at $P$ vacancy related defects. This explanation agrees well with the type of defects expected from the transmutation process where mainly points defects due to the $\gamma$ and $\beta^{-}$emissions should be generated. During the transmutation reaction of ${ }^{115} \mathrm{In}$ atoms, the average of the recoil energy transmitted to the involved atom through the $\beta^{-}$decay is close to $15 \mathrm{eV}$ which is enough to create vacancyinterstitial pairs in the In sublattice since only $5-8 \mathrm{eV}$ is required for the creation of Frenkel defects in III-V materials. Furthermore, the $\beta^{-}$decay is always accompanied by the emission of a cascade of 4 or $5 \gamma$ rays. The induced $\gamma$ recoils of the ${ }^{116}$ In (excited nuclei) occur with an average energy of $50 \mathrm{eV},{ }^{17}$ which confirms that the transmuted nuclei are come to rest not far away from their initial positions.
As the neutron absorption coefficient is 1000 times higher for In isotopes than for the $P$ nucleus, the probability to create a vacancy-related defect in the In-sublattice should be 1000 times higher than those created in the $P$ sublattice.

Concerning the defects create by the fast neutrons, it is expected to be similar to those created by ion implantation which consist mainly in clusters of about 50-100 defects. ${ }^{12}$ However, as the rapid neutron rate and the capture crosssection at this energies are low $(\sim \mathrm{b})$, one finds that the number of defects due to the $\beta$ recoil is very large compared with those created by the fast neutrons.

The three heaviest irradiated samples (10, 11, and 12) were annealed at $550{ }^{\circ} \mathrm{C}$ during $10 \mathrm{~min}$ and then measured at room temperature. Again, the spectra are well fitted with only one component. The lifetime values obtained for these samples have been plotted in Fig. 1.

The decrease observed in the lifetime shows that most of the positron traps produced during the transmutation process have been effectively annealed at this temperature.

As it can be seen in Fig. 1, the lifetime values for the annealed samples are slightly longer than the measured one on as-grown $\mathrm{InP}(246 \pm 2 \mathrm{ps})$ indicating the present of some remaining defects. This result can not be associated with the annealing time used (10 $\mathrm{min})$ because electrical measurements show that the samples exhibit the same electron concentration for different annealing times $(10 \mathrm{~s}, 10 \mathrm{~min}$ or $30 \mathrm{~s})$ at $550{ }^{\circ} \mathrm{C}$. ${ }^{9}$ The remaining defects are probably neutral since these only affect the low temperature mobility value and do not affect the carrier concentration. ${ }^{10}$

\section{CONCLUSIONS}

The evolution of the positron lifetime in NTD-InP crystals is presented. $\bar{\tau}$ shows a monotonous increase with the neutron dose. This effect shows that certain lattice defects, induced by thermal neutrons, act as effective positron traps. These defects are identified as indium vacancies impurities.

From thermal-annealing experiments at $550{ }^{\circ} \mathrm{C}$, a decrease of mean positron-lifetime is observed. In the samples doped above $[\mathrm{Sn}]=10^{17} \mathrm{~cm}^{-3}, \tau_{b}$ is not completely recovered suggesting that certain neutral defects remain in the crystal lattice.

\section{ACKNOWLEDGMENTS}

L. C. Damonte acknowledges the Comisión Interministerial de Ciencia y Tecnología for a postdoctoral fellowship during her stay at the I.F.I.C., Universidad de Valencia, Spain. F. J. Navarro acknowledges the Ministerio de Educación y Ciencia (Spain) for a FPI fellowship. This work was partly supported by the Ministerio de Educación y Ciencia (Project No. PB92-0098) and the Generalitat Valenciana (Project No. GV-3235/95).

\footnotetext{
${ }^{1}$ Topics in Current Physics, Vol. 12, edited by P. Hautojärvi (SpringerVerlag, Berlin).

${ }^{2}$ ICPA88, "Positron Annihilation," Proceedings of the 8th Internacional Conference on Positron Annihilation, Gent, 1988, edited by L. DorikensVanpraet, M. Dorikens, and D. Segers (World Scientific, Singapore, 1989).

${ }^{3}$ M. J. Puska and R. M. Nieminen, Rev. Modern Phys. 66, 841 (1994).

${ }^{4}$ S. Dannefaer, B. Hogg, and D. Kerr, Phys. Rev. B 30, 3355 (1984).
} 
${ }^{5}$ S. Dannefaer and D. Kerr, Phys. Rev. B 48, 9142 (1993).

${ }^{6}$ R. Ambigapathy, A. A. Manuel, P. Hautojärvi, K. Saarinen, and C. Corbel, Phys. Rev. B 50, 2188 (1994).

${ }^{7}$ G. Dlubek, O. Brümmer, F. Plazaola, P. Hautojärvi, and K. Naukarinen. Appl. Phys. Lett. 46, 1136 (1985).

${ }^{8}$ T. Bretagnon, S. Dannefaer, and D. Kerr, J. Appl. Phys. 73, 4697 (1993).

${ }^{9}$ B. Boudart, B. Mari, B. Prévot, and C. Schwab, Nucl. Instrum. Methods B 63, 101 (1992).

${ }^{10}$ B. Mari, B. Prevot, and C. Schwab, Mater. Sci. Eng. B 20, 113 (1993).

${ }^{11}$ Handbook of Chemistry and Physics, 73th ed., edited by R. Weast (CRC, Boca Raton, 1989).
${ }^{12}$ A. Goltzene, B. Meyer, and C. Schwab, J. Appl. Phys. 57, 1332 (1983).

${ }^{13}$ P. Kirkegaard and M. Eldrup, Comp. Phys. Commu. 3, 240 (1972).

${ }^{14}$ P. Kirkegaard and M. Eldrup, Comp. Phys. Commu. 7, 401 (1974).

${ }^{15}$ M. J. Puska, S. Mäkinen, M. Manninen, and R. J. Nieminen, Phys. Rev. B 39, 7666 (1989).

${ }^{16}$ C. Corbel, M. Stucky, P. Hautojärvi, K. Saarinen, and P. Moser, Phys. Rev. B 38, 8192 (1988).

${ }^{17}$ H. Grupp, K. Dör, H. J. Stöckmann, H. Ackermann, B. Bader, and W. Buttler, Z. Phys. B: Cond. Matter 47, 1 (1982). 